TITLE:

\title{
Identification of novel mammalian phospholipids containing threonine, aspartate, and glutamate as the base moiety.
}

\section{AUTHOR(S):}

Omori, Taketo; Honda, Ai; Mihara, Hisaaki;

Kurihara, Tatsuo; Esaki, Nobuyoshi

\section{CITATION:}

Omori, Taketo .... [et al]. Identification of novel mammalian phospholipids containing threonine, aspartate, and glutamate as the base moiety.. Journal of chromatography. B, Analytical technologies in the biomedical and life sciences 2011, 879(29): 3296-3302

\section{ISSUE DATE:}

2011-11-01

URL:

http://hdl.handle.net/2433/151862

\section{RIGHT:}

(C) 2011 Elsevier B.V.; This is not the published version. Please cite only the published version.; この論文は出版社版でありません。引用の際に は出版社版をご確認ご利用ください。 
Identification of novel mammalian phospholipids containing threonine, aspartate, and glutamate as the base moiety

Taketo Omori ${ }^{\mathrm{a}}$, Ai Honda ${ }^{\mathrm{a}}$, Hisaaki Mihara ${ }^{\mathrm{b}}$, Tatsuo Kurihara ${ }^{\mathrm{a}}$, and Nobuyoshi Esaki ${ }^{\mathrm{a}, *}$

${ }^{a}$ Institute for Chemical Research, Kyoto University, Uji, Kyoto

611-0011, Japan

${ }^{b}$ Department of Biotechnology, Institute of Science and Engineering,

College of Life Sciences, Ritsumeikan University, Kusatsu, Shiga 525-8577, Japan

*Corresponding author. 
Institute for Chemical Research, Kyoto University, Uji, Kyoto

611-0011, Japan

Tel.: +81-77-438-3240; Fax: +81-77-438-3248

E-mail: esakin@SCL.kyoto-u.ac.jp 


\section{Abstract}

Phosphatidylserine is a derivative of phosphatidic acid, in which a

phosphoric acid is bound by an ester linkage to the hydroxyl group of a

serine moiety. Recently, not only L-serine but also D-serine has been

shown to occur as a base moiety of the phospholipids found in various

rat tissues. In this study, we showed the occurrence of phosphatidyl-L-threonine (PThr), phosphatidyl-L-aspartate (PAsp), and phosphatidyl-L-glutamate (PGlu) in rat brain. Analyses using an HPLC-ESI-MS and an amino acid analyzer showed the presence of L-threonine, L-aspartate, and L-glutamate in the acid-hydrolysates of phospholipids from porcine cerebrum, rat cerebrum, and rat liver. Results of ESI-MS/MS analyses with neutral loss scanning and product ion scanning suggest the presence of PThr-(18:0, 18:1), PThr-(18:0, 
22:6), PAsp-(18:0, 18:1), PAsp-(18:0, 22:6), PGlu-(18:0, 18:1), and PGlu-(18:0, 22:6) in rat brain. This is the first study to identify 2 novel phospholipids, PAsp and PGlu, with a carboxylate-phosphate anhydride bond, in living organisms.

Keywords: aspartate, glutamate, threonine, phospholipids, mammal, carboxylate-phosphate anhydride

Abbreviations: Boc-L-Cys, N-tert-butyloxycarbonyl-L-cysteine; CEM, channel electron multiplier voltage; CM, carboxymethyl; DHA, docosahexaenoic acid; ESI, electrospray ionization; HPLC, high performance liquid chromatography; IS, ion spray voltage; MS, mass spectrometry; NMDA, $N$-methyl-D-aspartate; OPA, $o$-phthaldialdehyde; OR, orifice voltage; PAsp, phosphatidyl-L-aspartate; PGlu, 
phosphatidyl-L-glutamate; PS, phophatidylserine; L-PS,

phosphatidyl-L-serine; D-PS, phosphatidyl-D-serine; PThr,

phosphatidyl-L-threonine; RNG, focusing ring voltage; THF, tetrahydrofuran; TLC, thin-layer chromatography; 18:0, stearic acid;

18:1, oleic acid; 22:6, docosahexaenoic acid. 


\section{Introduction}

Phospholipids are the major lipid components of biological

membranes, in which sn-glycero-3-phosphate is esterified at its C1 and

C2 positions by fatty acids and at its phosphoryl group by various bases

such as choline, ethanolamine, and serine. Thus far, only 2 types of L-amino acid-containing phospholipids, i.e., phophatidylserine (PS) and phosphatidylthreonine (PThr), have been identified in biological samples. PS is a component of mammalian cell membranes and plays an important role in biological processes such as apoptosis [1-4] and cell signaling [5-8]. Although it was long believed that phosphatidyl-L-serine (L-PS) was the only PS occurring in living organisms [9-11], we recently discovered a D-serine-containing phospholipid, phosphatidyl-D-serine (D-PS), in rat cerebrum [12] and other tissues [13]. Meanwhile, PThr has 
been found in eggs [14], tuna muscle [15], polyoma virus-transformed hamster embryo fibroblasts [16], and hippocampal neurons cultured in a medium without serine [17]. However, it is still unclear whether PThr or D-PS plays any role in living organisms.

Both L-PS and PThr are synthesized by base-exchange reactions, in which the polar head groups of preexisting phospholipids are replaced by serine and threonine, respectively [17-20]. Similarly, D-PS has been suggested to be formed via a base-exchange mechanism, according to our previous finding of an endogenous activity through which free D-serine was incorporated into phospholipids from rat cerebrum extract [12]. This raises the question of whether other amino acids, besides serine and threonine, are incorporated as base moieties in phospholipids in vivo. In our previous studies [12, 13], we have noticed that threonine, aspartate, and glutamate are also present in the lipid hydrolysates of rat tissues. In 
this study, we analyzed the hydrolysates of porcine cerebrum by using HPLC-ESI-MS analysis and those of rat cerebrum and liver by using an amino acid analyzer. Through ESI-MS/MS analysis of the phospholipids from rat cerebrum, we showed that threonine, aspartate, and glutamate indeed occurred as the head groups of phospholipids found in rat cerebrum. To our knowledge, this is the first report of naturally occurring phospholipids possessing a carboxylate-phosphate anhydride bond.

\section{Materials and methods}

\subsection{Materials}

$o$-Phthaldialdehyde (OPA) and a Cosmosil 5 $\mathrm{C}_{18}$ - AR-II column (4.6 
$\times 150 \mathrm{~mm}$ ) were purchased from Nacalai Tesque (Kyoto, Japan).

$N$-tert-Butyloxycarbonyl-L-cysteine (Boc-L-Cys) was purchased from

Novabiochem (La Jolla, CA, USA). A preparative thin-layer

chromatography (TLC) plate (Silica Gel 60 glass plate) was purchased

from Merck (Darmstadt, Germany). A carboxymethyl (CM)-cellulose

column was purchased from Whatman International Ltd (Maidstone, UK).

All other chemicals were of analytical grade.

\subsection{Extraction and purification of lipids}

Crude lipids were extracted from porcine brain (1 g) by using

Folch's method [21]. To further purify the lipids, 0.2 volumes of $0.9 \%$

$\mathrm{KCl}$ was added to the crude lipid extract, and the mixture was

centrifuged at $100 \times g$ for $20 \mathrm{~min}$ at ambient temperature. The 
chloroform phase was collected and dried under a stream of nitrogen gas.

The purified lipids were resuspended in chloroform and stored at $-20^{\circ} \mathrm{C}$

until use.

Male Std:Wistar rats (Japan SLC, Inc., Japan) at postnatal week 7

were euthanized and their cerebrums were extirpated. The extraction and

purification of crude lipids were performed using the abovementioned procedures. PS-rich phospholipids were isolated from the purified lipids by preparative TLC by using the solvent system consisting of chloroform/methanol/acetic acid/water (65/25/2/4, v/v) [16] and by CM-cellulose column chromatography [22]. The purified phospholipids were stored at $-20^{\circ} \mathrm{C}$ until use. 
Phospholipids were hydrolyzed by boiling them in $1 \mathrm{ml}$ of $6 \mathrm{M} \mathrm{HCl}$

for $6 \mathrm{~h}$. The hydrolysate was mixed with 9 volumes of chloroform/methanol/water $(27: 13: 5, \mathrm{v} / \mathrm{v})$ and centrifuged at $100 \times \mathrm{g}$ for $20 \mathrm{~min}$. The aqueous fraction containing amino acids was collected and stored at $-20^{\circ} \mathrm{C}$ until use.

2.4. Amino acid analysis

The aqueous fraction was applied to a Hitachi L-8500 amino acid analyzer equipped with a packed column for physiological fluid analysis

(Hitachi Ltd., Tokyo, Japan).

\subsection{Derivatization of amino acids}


Amino acids were derivatized using OPA and Boc-L-Cys according

to the method described by Hashimoto et al. [23].

2.6. HPLC-ESI-MS analysis

Boc-L-Cys-OPA amino acid derivatives were separated using an

HPLC system (Shimadzu, Kyoto, Japan) equipped with a Cosmosil

$5 \mathrm{C}_{18}$-AR-II column $(4.6 \times 250 \mathrm{~mm})$ operated at $0.8 \mathrm{ml} / \mathrm{min}$ at $20^{\circ} \mathrm{C}$ with a linear gradient from $20 \%$ to $40 \%$ mobile phase B over a period of $20 \mathrm{~min}$.

Mobile phase A consisted of $0.1 \mathrm{M}$ acetate buffer ( $\mathrm{pH}$ 6.0), $7 \%$ acetonitrile, and 3\% tetrahydrofuran (THF), and mobile phase B consisted of $0.1 \mathrm{M}$ acetate buffer ( $\mathrm{pH} 6.0$ ), $47 \%$ acetonitrile, and $3 \%$ THF, according to previously described methods [23]. The flow rate of the eluent from the HPLC column was split into a ratio of $1: 16$ such that 
only $50 \mu 1 / \mathrm{min}$ of the eluent entered the mass spectrometer. The mass spectrometric measurements were performed using an API 3000 triple quadrupole instrument (PE Sciex, Toronto, Canada). To measure the negative ion spectra, we set the ion spray voltage (IS), orifice voltage (OR), focusing ring voltage (RNG), and Channel Electron Multiplier voltage $(C E M)$ at $-4.0 \mathrm{kV},-30 \mathrm{~V},-200 \mathrm{~V}$, and $2.2 \mathrm{kV}$, respectively. The instrument was calibrated using a polypropylene glycol solution. Nitrogen was used as the nebulizer gas, curtain gas, and collision gas. The data were acquired over the mass ranges of 421-424, 435-437, $450-454$, and $463-467 \mathrm{~m} / \mathrm{z}$.

2.7. ESI-MS/MS analysis

Purified phospholipids in chloroform were dried under a stream of 
nitrogen gas and resuspended in acetonitrile/methanol (2/1, v/v)

containing $0.1 \%$ ammonium formate $(\mathrm{pH}$ 6.5). The lipid solution was

injected into the ESI-MS/MS system (PE Sciex, Toronto, Canada) and analyzed using the neutral loss scan and product ion scan modes. In both the scan modes, IS, OR, RNG, CEM, and collision energy were set at $-5.0 \mathrm{kV},-80 \mathrm{~V},-200 \mathrm{~V}, 2.8 \mathrm{kV}$, and $51 \mathrm{eV}$, respectively. A syringe infusion pump (Harvard Apparatus, Cambridge, MA) delivered solvent at $2.5 \mu \mathrm{l} / \mathrm{min}$ to the ESI probe. The lipid solution was introduced using a 100- $\mu$ l glass syringe with a stainless steel needle (Hamilton Co., Reno, $\mathrm{NV}$.

\section{Results}

\subsection{Identification of amino acids in a hydrolysate of lipids from porcine}


cerebrum

To investigate whether amino acids other than serine can exist as

the base moiety of phospholipids, lipids from porcine cerebrum were

hydrolyzed with $\mathrm{HCl}$. The hydrolysates were derivatized using OPA and

Boc-L-Cys [23] and subjected to HPLC-ESI-MS analysis. As shown in

Fig. 1A, 3 peaks were detected at $6.86,8.74$, and $13.82 \mathrm{~min}$ in addition

to those representing derivatives of $\mathrm{D}$-serine $(12.72 \mathrm{~min})$ and $\mathrm{L}$-serine

(11.76 $\mathrm{min}$ ). The $\mathrm{m} / \mathrm{z}$ values of the peaks at $6.86,8.74$, and $13.82 \mathrm{~min}$

were 451,465 , and 437 , respectively, which matched the $\mathrm{m} / \mathrm{z}$ values of

the Boc-L-Cys-OPA derivatives of authentic L-aspartate, L-glutamate,

and L-threonine, respectively. The retention times of the 3 peaks were

also consistent with those of the authentic derivatives of L-aspartate,

L-glutamate, and L-threonine (data not shown). The 3 peaks were not 
detected in the lipid samples that were boiled in water instead of $\mathrm{HCl}$

(Fig. 1B), and this finding confirmed that the lipid samples were not contaminated by free amino acids. These results suggest that L-aspartate, L-glutamate, and L-threonine occur as base moieties of phospholipids in porcine cerebrum.

\subsection{Analysis of phospholipid hydrolysates from rat cerebrum and liver}

To further examine whether aspartate, glutamate, and threonine can be liberated from phospholipids by hydrolysis, we extracted lipids from rat liver and isolated a PS-rich phospholipid fraction by using TLC and CM cellulose column chromatography. Because the mobility of PThr and PS is indistinguishable on TLC plates and CM cellulose columns [16], it was expected that the PS-rich phospholipid fraction would at 
least contain PThr. The purified PS-rich fraction was hydrolyzed by $\mathrm{HCl}$ and analyzed using an amino acid analyzer. As shown in Fig. 2A, peaks corresponding to not only serine and threonine but also aspartate and glutamate were detected. We also similarly analyzed the hydrolysate of a PS-rich fraction obtained from rat cerebrum and detected aspartate, glutamate, and threonine in addition to serine as the products of phospholipid hydrolysis (Fig. 2B). These results strongly suggest that not only serine and threonine but also aspartate and glutamate exist as acid-hydrolyzable moieties of phospholipids in the cerebrum and liver of rats. Our data also suggest that the mobility of phospholipids containing aspartate and glutamate is similar to that of PS and PThr under TLC and CM-cellulose column chromatography conditions.

\subsection{ESI-MS/MS analyses of phospholipids from rat cerebrum}


According to the results described above, if aspartate and

glutamate are bound to phosphatidic acid to form carboxylate-phosphate

anhydride, then phosphatidyl-L-aspartate (PAsp) and

phosphatidyl-L-glutamate (PGlu) are expected to occur in rat tissues (Fig.

3). To confirm this hypothesis, we analyzed the phospholipids found in

rat brain by performing fragmentation reactions of the polar head groups

of phospholipids during ESI-MS/MS. Fragmentation reactions allow

specific detection of a phospholipid species by neutral loss scanning.

Through this technique, it was shown that PS and PThr can be selectively

detected by a neutral loss of 87 Da [24, 25] and 101 Da [26],

respectively (Scheme. 1). Accordingly, when similar fragmentation

reactions occur in PAsp and PGlu, it is expected that neutral losses of

$115 \mathrm{Da}$ and $129 \mathrm{Da}$, respectively, should be observed. As expected, our 
ESI-MS/MS analysis of phospholipids found in rat brain detected neutral loss peaks of $115 \mathrm{Da}$ and $129 \mathrm{Da}$ (Fig. 4B and 4C). The neutral loss peak of $101 \mathrm{Da}$, corresponding to PThr, was also detected (Fig. 4A). These data suggest that PAsp and PGlu as well as PThr do occur in fractions of rat brain phospholipids.

Furthermore, we performed product ion scanning of the following peaks, identified as major peaks by the neutral loss scan: $m / z 802.7$ and 848.7 corresponding to PThr (Fig. 5A and 5B), $m / z \quad 816.7$ and 862.7 corresponding to PAsp (Fig. 5C and 5D), and $m / z \quad 830.3$ and 876.8 corresponding to PGlu (Fig. 5E and 5F). The product ion scans detected several peaks that can be assigned to fatty acid fragments (Fig. 5). The fragments at $m / z 281.5,283.5$, and 327.5 were identified as the anions of oleic acid, stearic acid, and docosahexaenoic acid (DHA), respectively. The fragment at $m / z \quad 153$ was consistent with the 1,2-cyclic 
phosphodiester of glycerol, which is a product ion from every glycerophospholipid [24, 25]. These results suggest the presence of PThr-(18:0, 18:1), PThr-(18:0, 22:6), PAsp-(18:0, 18:1), PAsp-(18:0, 22:6), PGlu-(18:0,18:1), and PGlu-(18:0, 22:6) in rat brain.

\section{Discussion}

We previously showed that D-PS occurs in rat cerebrum and various other rat tissues. We also showed that free D-serine can be incorporated into PS in an in vitro system by using cerebrum extract, suggesting that D-PS is formed by a base-exchange reaction $[12,13]$. In mammals, free D-serine is distributed in the cerebrum [27, 28] and plays an important role as a coagonist of the glycine site of $N$-methyl-D-aspartate (NMDA) receptors $[29,30]$. The intracellular 
D-serine levels are regulated by serine racemase $[31,32]$ and $D$-amino acid oxidase with its effector pLG72 [33]. In addition, PS is an abundant component of membranes of brain cells, and thus, our previous results suggest that the synthesis and degradation of D-PS affects the intracellular D-serine levels, thereby modulating neurotransmission.

In this study, we found that amino acids other than D- and L-serine occur as the base moieties of phospholipids in porcine and rat brains. From our data, these amino acids were determined to be L-threonine, L-aspartate, and L-glutamate. L-Threonine, in addition to serine, possesses a hydroxyl group in its side chain and thus probably esterifies at the phosphoryl group of a $s n$-glycero-3-phosphate phospholipid backbone. In contrast, the side chains of L-aspartate and L-glutamate contain a carboxylate group. Therefore, we propose that these dicarboxylic amino acids form a carboxylate-phosphate anhydride bond 
with phosphatidic acid. To our knowledge, this is the first study that showed the presence of phospholipids with a carboxylate-phosphate anhydride bond. No other amino acids, except serine, threonine, aspartate, and glutamate, were detected in our HPLC-ESI-MS and amino acid analyses. Especially, hydroxyl-containing tyrosine and homoserine and reactive thiol-containing cysteine and homocysteine were not detected in the lipid hydrolysates. This finding indicates that PThr, PAsp, and PGlu are not nonspecific resultant products, which could have been indistinguishably formed by the base-exchange reactions of phospholipids. Instead, they are possibly synthesized by particular metabolic reactions. Although a wide variety of $\alpha$-carboxyl group-containing amino acids exists, only L-aspartate and L-glutamate, in addition to L-threonine and L- and D-serine were found in the lipid hydrolysates. Therefore, we propose that the carboxylate-phosphate 
anhydride bond in PAsp and PGlu is formed between the phosphoryl group of a phospholipid and the distal carboxyl groups (i.e., $\beta$-carboxyl and $\gamma$-carboxyl) of L-aspartate and L-glutamate. Unlike D-PS, D-aspartate was not found in the hydrolysate of phospholipids despite the fact that a considerable amount of D-aspartate occurs in various endocrine tissues $[28,34,35]$ to regulate production and/or secretion of specific hormones $[36,37]$. However, this is not unexpected because Hashimoto et al. reported that significant considerable amount of free D-aspartate is not present in rat cerebrum at postnatal week 7 [28]. It will be interesting to analyze hydrolysates of phospholipids from testis, which contain relatively high concentrations of free D-aspartate [28], to examine the presence of phosphatidyl-D-aspartate.

It has already been reported that PThr occurs in several mammalian cells, fish, and eggs [14-17]. Although the physiological 
function of PThr remains unknown, its putative metabolic pathway has been suggested. It is thought that PThr can be synthesized by a base-exchange enzyme that also catalyzes the synthesis of PS [17] and can be decarboxylated to yield phosphatidylisopropanolamine by PS decarboxylase [26]. It has been proposed that PThr is synthesized instead of PS when the intracellular levels of serine are insufficient for the synthesis of PS [17]. However, the results of the present study suggest that PThr appears to be constitutively present in both normal rat brain and normal porcine liver. This implies an as of yet unknown biological role played by PThr in mammals.

On the other hand, the metabolic pathway of PAsp and PGlu remains unclear. There is no reported evidence that PS synthase or PS decarboxylase acts on phospholipids possessing a carboxylate-phosphate anhydride bond. This raises an interesting question about the metabolism 
of phospholipids. It is known that aspartate and glutamate act as neurotransmitters involved in learning and memory in mammalian brains [38, 39]. Thus, PAsp and PGlu are potentially related to these neural processes through regulation of the brain levels of free aspartate and glutamate.

Our MS/MS analyses showed that PThr, PAsp, and PGlu each contain either a set of oleic acid and stearic acid or a set of oleic acid and DHA as the fatty acid moieties. Polyunsaturated fatty acids play essential roles in the development and function of the brain [40-42]. In particular, decreased levels of DHA in the brain and retina are associated with impairments in neural and visual functions [43-45]. PS contains a high proportion (>35\%) of DHA as a fatty acid moiety [46]. It is thought that the developmental disorder of the central nervous system observed in fetal alcohol syndrome is partly due to a decreased level of 
DHA-containing PS [47-50]. Further studies are needed to investigate the potential biological roles played by the DHA-containing phospholipids discovered in this study.

\section{Conclusions}

We investigated the base moieties of phospholipids found in rat brain and liver and porcine cerebrum by a combination of HPLC-ESI-MS analysis, amino acid analysis, and ESI-MS/MS analysis followed by neutral loss scanning and product ion scanning. Our data suggest the presence of PThr-(18:0, 18:1), PThr-(18:0, 22:6), PAsp-(18:0, 18:1), PAsp-(18:0, 22:6), PGlu-(18:0, 18:1), and PGlu-(18:0, 22:6) in mammalian tissues. This study is the first to identify naturally occurring phospholipids with carboxylate-phosphate anhydride bonds. 


\section{References}

[1] V.A. Fadok, D.R. Voelker, P.A. Campbell, J.I. Cohen, D.L. Bratton,

P.M. Henson, J. Immunol. 148 (1992) 2207.

[2] V.A. Fadok, A. de Cathelineau, D.L. Daleke, P.M. Henson, D.L.

Bratton, J. Biol. Chem. 276 (2001) 1071.

[3] M.O. Li, M.R. Sarkisian, W.Z. Mehal, P. Rakic, R.A. Flavell, Science $302(2003) 1560$.

[4] K. Balasubramanian, B. Mirnikjoo, A.J. Schroit, J. Biol. Chem. 282 (2007) 18357.

[5] Y. Nishizuka, Nature 308 (1984) 693.

[6] R.M. Bell, D.J. Burns, J. Biol. Chem. 266 (1991) 4661.

[7] Y. Nishizuka, Science 258 (1992) 607.

[8] L. Bittova, R.V. Stahelin, W. Cho, J. Biol. Chem. 276 (2001) 4218. 
[9] J. Folch, H.A. Schneider, J. Biol. Chem. 137 (1941) 51.

[10] J. Folch, J. Biol. Chem. 139 (1941) 973.

[11] J. Folch, J. Biol. Chem. 174 (1947) 439.

[12] T. Omori, H. Mihara, T. Kurihara, N. Esaki, Biochem. Biophys. Res.

Commun. 382 (2009) 415.

[13] T. Omori, H. Mihara, T. Kurihara, N. Esaki, Biosci. Biotechnol.

Biochem. 74 (2010) 1953.

[14] D.N. Rhodes, C.H. Lea, Biochem. J. 65 (1957) 526.

[15] H. Igarashi, K. Zama, M. Katada, Nature 181 (1958) 1282.

[16] D. Mark-Malchoff, G.V. Marinetti, G.D. Hare, A. Meisler, Biochemistry 17 (1978) 2684.

[17] J. Mitoma, T. Kasama, S. Furuya, Y. Hirabayashi, J. Biol. Chem. 273 (1998) 19363.

[18] O. Kuge, M. Nishijima, Y. Akamatsu, J. Biol. Chem. 266 (1991) 
24184.

[19] O. Kuge, K. Saito, M. Nishijima, J. Biol. Chem. 272 (1997) 19133.

[20] J.E. Vance, R. Steenbergen, Prog. Lipid Res. 44 (2005) 207.

[21] J. Folch, I. Ascoli, M. Lees, J.A. Meath, N. Le Baron, J. Biol. Chem.

$191(1951) 833$.

[22] P. Comfurius, R.F. Zwaal, Biochim. Biophys. Acta 488 (1977) 36.

[23] A. Hashimoto, T. Nishikawa, T. Oka, K. Takahashi, T. Hayashi, J.

Chromatogr. 582 (1992) 41.

[24] B. Brugger, G. Erben, R. Sandhoff, F.T. Wieland, W.D. Lehmann,

Proc. Natl. Acad. Sci. USA 94 (1997) 2339.

[25] M. Pulfer, R.C. Murphy, Mass Spectrom. Rev. 22 (2003) 332.

[26] L. Heikinheimo, P. Somerharju, Traffic. 3 (2002) 367.

[27] A. Hashimoto, T. Nishikawa, T. Hayashi, N. Fujii, K. Harada, T.

Oka, K. Takahashi, FEBS Lett. 296 (1992) 33. 
[28] A. Hashimoto, T. Oka, T. Nishikawa, Eur. J. Neurosci. 7 (1995) 1657.

[29] A. Hashimoto, T. Nishijima, T. Oka, K. Takahashi, J. Neurochem. 60 (1993) 783.

[30] M.J. Schell, M.E. Molliver, S.H. Snyder, Proc. Natl. Acad. Sci. USA 92 (1995) 3948.

[31] H. Wolosker, K.N. Sheth, M. Takahashi, J.P. Mothet, R.O. Brady Jr., C.D. Ferris, S.H. Snyder, Proc. Natl. Acad. Sci. USA 96 (1999) 721.

[32] V.N. Foltyn, I. Bendikov, J. De Miranda, R. Panizzutti, E. Dumin, M.

Shleper, P. Li, M.D. Toney, E. Kartvelishvily, H. Wolosker, J. Biol.

Chem. 280 (2005) 1754.

[33] S. Sacchi, M. Bernasconi, M. Martineau, J.P. Mothet, M. Ruzzene, M.S. Pilone, L. Pollegioni, G. Molla, J. Biol. Chem. 283 (2008) 22244.

[34] K. Imai, T. Fukushima, T. Oka, K. Takahashi, Biomed Chromatogr. 
$9(1995) 106$.

[35] T. Furuchi, H. Homma, Biol. Pharm. Bull. 28 (2005) 1566.

[36] Y. Takigawa, H. Homma, J.A. Lee, T. Fukushima, T. Santa, T.

Iwatsubo, K. Imai, Biochem. Biophys. Res. Commun. 248 (1998) 641.

[37] Y. Nagata, H. Homma, J.A. Lee, K. Imai, FEBS Lett. 444 (1999)

160.

[38] P.R. Maycox, J.W. Hell, R. Jahn, Trends Neurosci. 13 (1990) 83.

[39] T. Miyaji, N. Echigo, M. Hiasa, S. Senoh, H. Omote, Y. Moriyama, Proc. Natl. Acad. Sci. USA 105 (2008) 11720.

[40] M. Vreugdenhil, C. Bruehl, R.A. Voskuyl, J.X. Kang, A. Leaf, W.J.

Wadman, Proc. Natl. Acad. Sci. USA 93 (1996) 12559.

[41] K. Kitajka, L.G. Puskas, A. Zvara, L. Hackler, Jr., G. Barcelo-Coblijn, Y.K. Yeo, T. Farkas, Proc. Natl. Acad. Sci. USA 99 (2002) 2619. 
[42] K. Kitajka, A.J. Sinclair, R.S. Weisinger, H.S. Weisinger, M. Mathai, A.P. Jayasooriya, J.E. Halver, L.G. Puskas, Proc. Natl. Acad. Sci. USA $101(2004) 10931$.

[43] R. Uauy, D.R. Hoffman, P. Peirano, D.G. Birch, E.E. Birch, Lipids $36(2001) 885$.

[44] J. Catalan, T. Moriguchi, B. Slotnick, M. Murthy, R.S. Greiner, N.

Salem, Jr., Behav. Neurosci. 116 (2002) 1022.

[45] L.G. Puskas, E. Bereczki, M. Santha, L. Vigh, G. Csanadi, F. Spener, P. Ferdinandy, A. Onochy, K. Kitajka, Biochim. 86 (2004) 817.

[46] R. Hitzemann, Neurochem. Res. 6 (1981) 935.

[47] P.S. Sastry, Prog. Lipid. Res. 24 (1985) 69.

[48] R.A. Harris, D.M. Baxter, M.A. Mitchell, R.J. Hitzemann, Mol. Pharmacol. 25 (1984) 401.

[49] Z. Wen, H.Y. Kim, J. Neurochem. 89 (2004) 1368. 
[50] Z. Wen, H.Y. Kim, J. Neurosci. Res. 85 (2007) 1568. 


\section{Figure captions}

Fig. 1. HPLC-ESI-MS analysis of the acid-hydrolysates of lipids from porcine brain.

(A) PS was hydrolyzed using $6 \mathrm{M} \mathrm{HCl}$ for $6 \mathrm{~h}$. Free amino acids were isolated and derivatized using Boc-L-Cys and OPA. (B) PS was boiled in water instead of $\mathrm{HCl}$. The aqueous fraction was isolated and applied to the derivatization procedure. Data were acquired over the mass ranges of $421-424,435-437,450-454$, and 463-467 m/z. The molecular masses of serine, threonine, aspartate, and glutamate derivatives were 424, 438, 452, and 466 Da, respectively. The peaks of the Boc-L-Cys-OPA derivatives of L-aspartate, L-glutamate, L-threonine, L-serine, and D-serine are indicated by $a, b, c, d$, and $e$, respectively. 
Fig. 2. Amino acid analysis of the acid-hydrolysates of lipids found in rat cerebrum and liver.

A chromatogram of amino acids detected in a hydrolysate of rat liver phospholipids (A) and rat cerebrum phospholipids (B), which were purified by preparative TLC and CM-cellulose column chromatography, is shown. The peaks representing aspartate, threonine, glutamate, and serine are indicated by $a, b, c$, and $d$, respectively.

Fig. 3. Structures of PS, PThr, PAsp, and PGlu.

The known structures of PS (A) and PThr (B) and the proposed structures of PAsp (C) and PGlu (D) are shown.

Fig. 4. ESI-MS/MS analysis with neutral loss scanning of the phospholipids detected in rat brain. 
Data of mass spectrometric analyses are shown: PThr with a neutral loss of 101 Da (A), PAsp with a neutral loss of 115 Da (B), and PGlu with a neutral loss of 129 Da (C). The inset of each panel shows the assumed fragmentation reaction in ESI-MS/MS.

Fig. 5. ESI-MS/MS analysis with product ion scanning of the phospholipids found in rat brain.

Product ion spectra of each $[\mathrm{M}-\mathrm{H}]^{-}$ion, indicated in Fig. 3, with a molecular mass of 802.7 Da (A), 848.7 Da (B), 816.7 Da (C), 862.7 Da (D), 830.3 Da (E), and 876.8 Da (F), are shown. The peaks corresponding to a glycerol phosphate fragment, stearic acid (18:0), oleic acid (18:1), and docosahexaenoic acid (22:6) are also indicated.

Scheme. 1 Analysis of the fragmentation reactions of PS and PThr in 
ESI-MS/MS. 
A

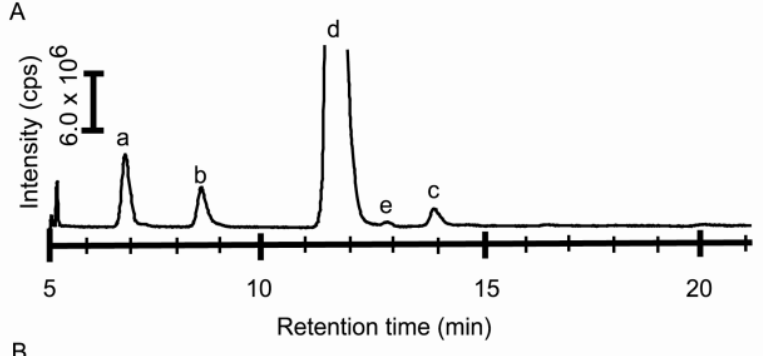

B

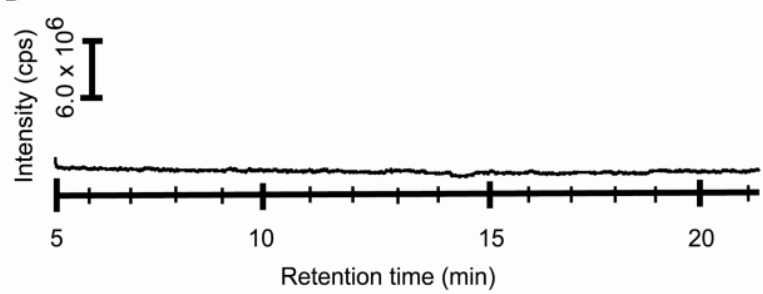

Fig. 1 T. Omori et al. 

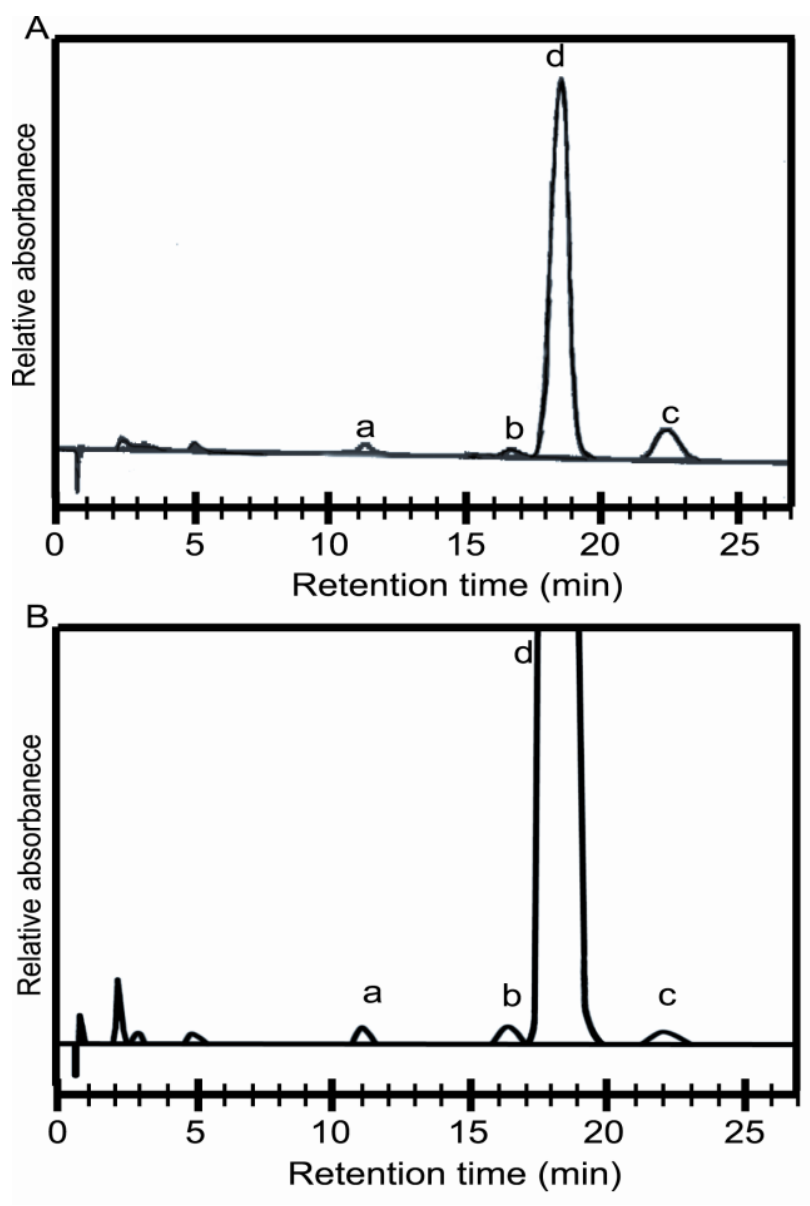

Fig. 2 T. Omori et al. 


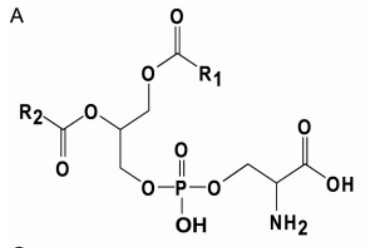

C

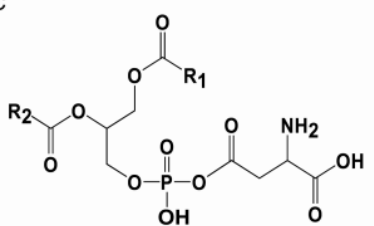

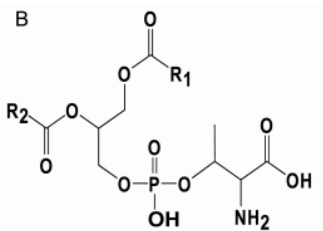

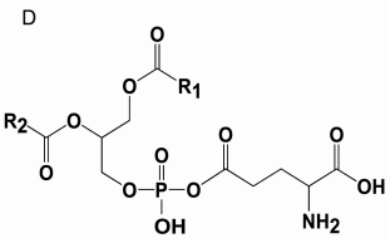

Fig. 3 T. Omori et al. 


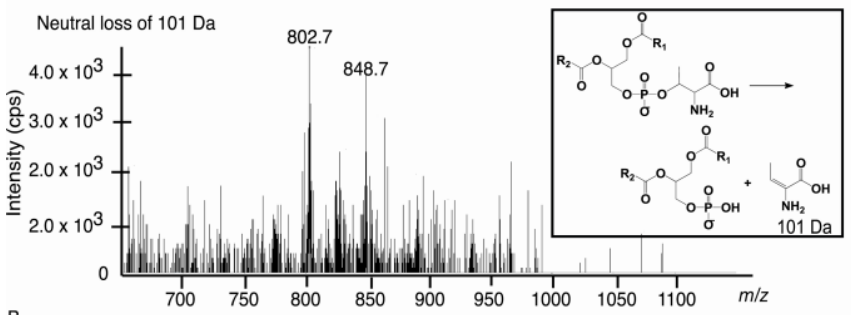

B Neutral loss of $115 \mathrm{Da}$

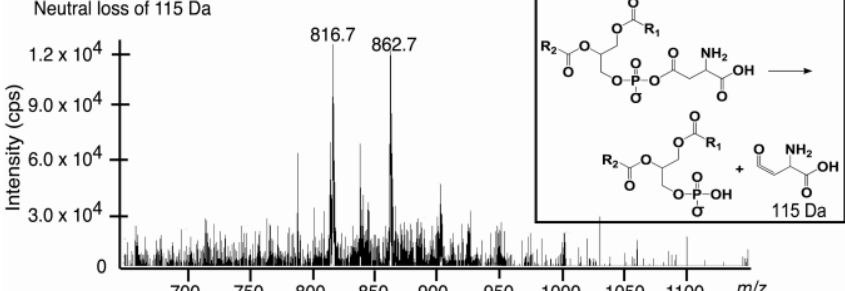

c

$\begin{array}{llllllllll}700 & 750 & 800 & 850 & 900 & 950 & 1000 & 1050 & 1100 & \mathrm{~m} / \mathrm{z}\end{array}$

C Neutral loss of $129 \mathrm{Da}$

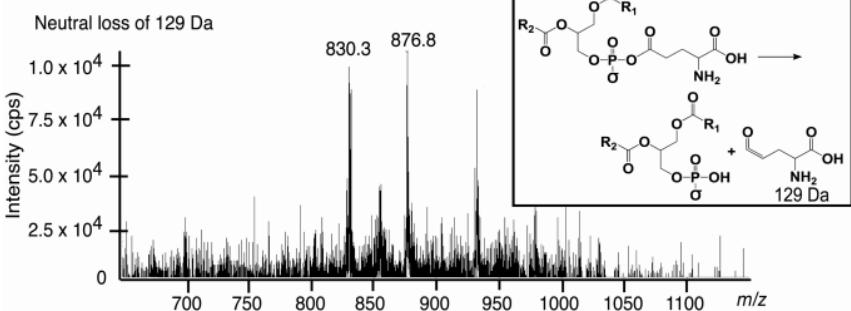

Fig.4 T. Omori et al. 

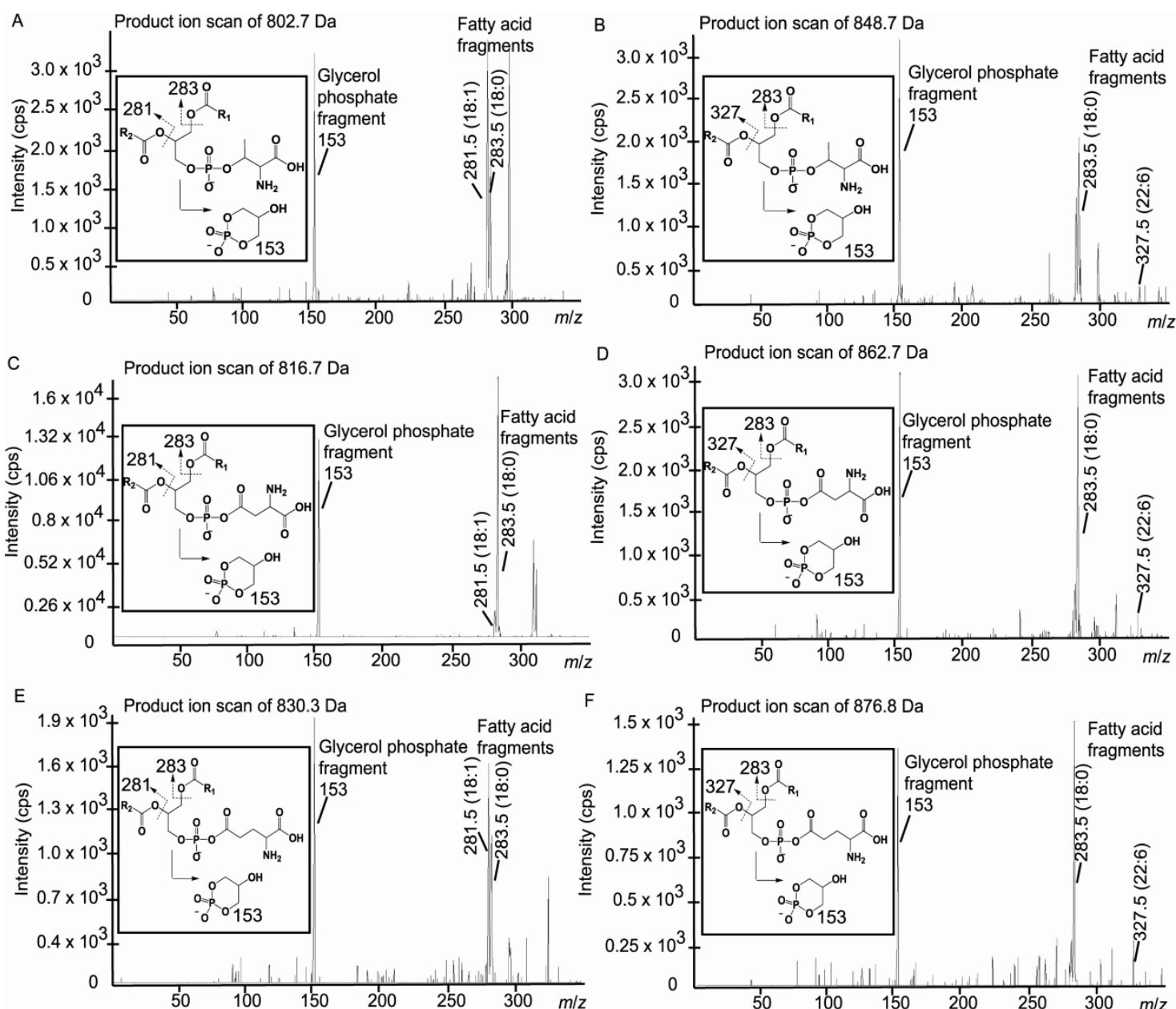

Fig.5 T. Omori et al. 

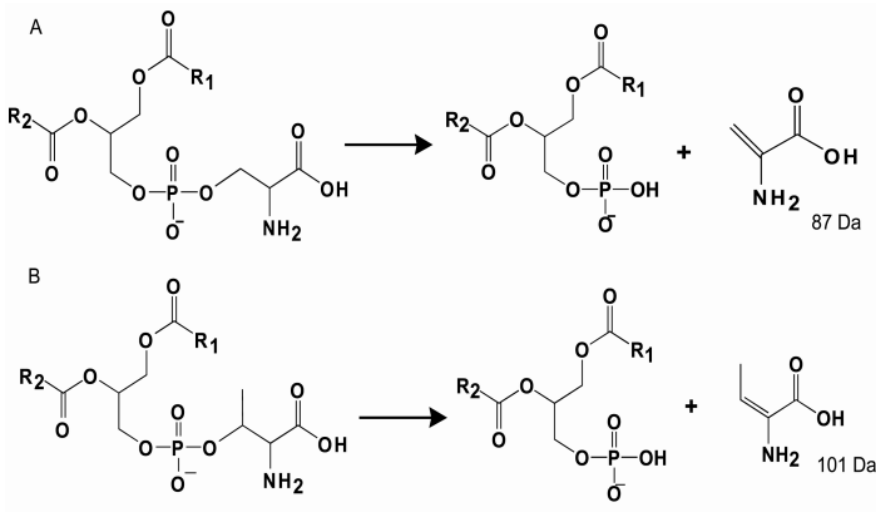

Scheme 1 T. Omori et al. 